\title{
Thiabendazole inhibits ubiquinone reduction activity of mitochondrial respiratory complex II via a water molecule mediated binding feature
}

\author{
Qiangjun Zhou ${ }^{1,2}$, Yujia Zhai ${ }^{1}$, Jizhong Lou ${ }^{1}$, Man Liu ${ }^{1}$, Xiaoyun Pang ${ }^{1}$, Fei Sun ${ }^{1 凶}$ \\ ${ }^{1}$ National Laboratory of Biomacromolecules, Institute of Biophysics, Chinese Academy of Sciences, Beijing 100101, China \\ ${ }^{2}$ Graduate School of the Chinese Academy of Sciences, Beijing 100080, China \\ Correspondence: feisun@ibp.ac.cn
}

Received June 29, 2011 Accepted July 19, 2011

\begin{abstract}
The mitochondrial respiratory complex II or succinate: ubiquinone oxidoreductase (SQR) is a key membrane complex in both the tricarboxylic acid cycle and aerobic respiration. Five disinfectant compounds were investigated with their potent inhibition effects on the ubiquinone reduction activity of the porcine mitochondrial SQR by enzymatic assay and crystallography. Crystal structure of the SQR bound with thiabendazole (TBZ) reveals a different inhibitor-binding feature at the ubiquinone binding site where a water molecule plays an important role. The obvious inhibitory effect of TBZ based on the biochemical data $\left(\mathrm{IC}_{50} \sim 100 \mu \mathrm{mol} / \mathrm{L}\right)$ and the significant structure-based binding affinity calculation ( $\sim 94 \mu \mathrm{mol} / \mathrm{L})$ draw the suspicion of using TBZ as a good disinfectant compound for nematode infections treatment and fruit storage.
\end{abstract}

KEYWORDS mitochondrial respiratory complex II, thiabendazole, pentachlorophenol, inhibition, crystallography

\section{INTRODUCTION}

Mitochondria are cellular organelles of prokaryotic origin that are found in almost all eukaryotic cells. The mitochondrial respiratory system, consisting of five membrane protein complexes (I to $\mathrm{V}$ ), produces most of the energy in eukaryotic cells by a process called oxidative phosphorylation (Saraste, 1999). Mitochondrial Complex II (Yankovskaya et al., 2003; Sun et al., 2005; Huang et al., 2006), also known as mitochondrial succinate:ubiquinone oxidoreductase (SQR) (EC 1.3.5.1), is a key membrane complex in the tricarboxylic acid cycle (Krebs cycle) that catalyzes the oxidation of succinate to fumarate in the mitochondrial matrix as succinate dehydrogenase. Succinate oxidation is coupled to reduction of ubiquinone to ubiquinol at the mitochondrial inner membrane as one part of the electron transfer chain. Electrons are transferred from succinate to ubiquinone through the buried prosthetic groups flavin-adenine dinucleotide (FAD), [2Fe-2S], [4Fe-4S], [3Fe-4S] clusters and heme, which form an integral part of SQR (Hägerhäll, 1997). Mitochondrial SQR contains four non-identical subunits: the FAD-binding protein or flavoprotein $(\mathrm{Fp}, 68 \mathrm{kDa})$, the iron-sulfur protein ( $\mathrm{lp}, 29 \mathrm{kDa}$ ), and two membrane anchor proteins (CybL, $15 \mathrm{kDa}$, and CybS, $11 \mathrm{kDa}$ ) with a total of six transmembrane helices and the heme cofactor embedded in. $S Q R$ is closely related to quinol:fumarate oxidoreductase (QFR, also called fumarate reductase, FRD) that catalyses the reverse reaction in anaerobic respiratory chains using fumarate as the terminal electron acceptor.

Several crystal structures have been obtained for QFR and SQR from Escherichia coli (Iverson et al., 1999; Yankovskaya et al., 2003), QFR from Wolinella succinogenes (Lancaster et al., 1999) and SQR from porcine (Sun et al., 2005) and avian (Huang et al., 2006). These structures revealed the overall similar architectures, the ubiquitous arrangement of the subunits, the positions of buried prosthetic groups, the electron transfer pathway and the ubiquinone-binding sites. The ubiquinone binding sites (Q-sites) were further confirmed by structures of QFR/SQR bound with the ubiquinone reduction inhibitors (also called Q-site inhibitors) (Table 1). Although SQR and QFR have the same ancestor and share similar architectures, their Q-sites were compared and showed obvious differences (Cecchini et al., 2003; Horsefield et al., 2006; Maklashina and Cecchini, 2010). As a result, 
Table 1 The Q -site inhibitors from the reported complex structures with SQR or QFR

\begin{tabular}{|c|c|c|c|c|c|}
\hline Name & Abbreviation & Chemical name & PDB & Resolution $(\AA)$ & Complex name \\
\hline \multirow[t]{2}{*}{ Carboxin } & \multirow[t]{2}{*}{ CBX } & \multirow[t]{2}{*}{$\begin{array}{l}\text { 2-methyl- } N \text {-phenyl-5,6- } \\
\text { 1,4-oxathiine-3-carboxamide }\end{array}$} & $2 \mathrm{WDQ}$ & 2.4 & $\begin{array}{l}\text { E. coli SQR bound with CBX } \\
\text { (Ruprecht et al., 2009) }\end{array}$ \\
\hline & & & $2 \mathrm{FBW}$ & 2.1 & $\begin{array}{l}\text { Avian mitochondrial SQR } \\
\text { bound with CBX (Huang et } \\
\text { al., 2006) }\end{array}$ \\
\hline Atpenin A5 & AA5 & $\begin{array}{l}\text { 3-[(2S,4S,5R)-5,6-Dichloro- } \\
\text { 2,4-dimethyl-1-oxohexyl]- } \\
\text { 4-hydroxy-5,6-dimethoxy- } \\
\text { 2(1H)-Pyridinone }\end{array}$ & $2 A C Z$ & 3.1 & $\begin{array}{l}\text { E. coli SQR bound with AA5 } \\
\text { (Horsefield et al., 2006) }\end{array}$ \\
\hline 2-thenoyltrifluoroacetone & TTFA & $\begin{array}{l}\text { 4,4,4-trifluoro-1-(2-thienyl)- } \\
\text { 1,3-butanedione }\end{array}$ & $1 \mathrm{ZP0}$ & 3.5 & $\begin{array}{l}\text { Porcine mitochondrial SQR } \\
\text { bound with 3-nitropropionate } \\
\text { and TTFA (Sun et al., 2005) }\end{array}$ \\
\hline Dinitrophenol-17 & DNP-17 & $\begin{array}{l}\text { 2-(1-methylhexyl)-4,6- } \\
\text { dinitrophenol }\end{array}$ & 1NEN & 2.9 & $\begin{array}{l}\text { E. coli SQR bound with DNP- } \\
17 \text { (Yankovskaya et al., 2003) }\end{array}$ \\
\hline Pentachlorophenol & PCP & $\begin{array}{l}\text { 1-hydroxypentachloroben- } \\
\text { zene }\end{array}$ & $2 \mathrm{WDR}$ & 3.2 & $\begin{array}{l}\text { E. coli SQR bound with PCP } \\
\text { (Ruprecht et al., 2009) }\end{array}$ \\
\hline Dinitrophenol-19 & DNP-19 & $\begin{array}{l}\text { 2-[1-(p-chlorophenyl)ethyl] } \\
\text { 4,6-dinitrophenol }\end{array}$ & $1 \mathrm{KFY}$ & 3.6 & $\begin{array}{l}\text { E. coli QFR bound with DNP- } \\
19 \text { (Iverson et al., 2002) }\end{array}$ \\
\hline \multicolumn{2}{|c|}{$\begin{array}{l}\text { 2-heptyl-4-hydroxyquinoline- HQNO } \\
\text { N-oxide }\end{array}$} & $\begin{array}{l}\text { 2-heptyl-4-hydroxyquinoline- } \\
\text { N-oxide }\end{array}$ & $1 \mathrm{KF} 6$ & 2.7 & $\begin{array}{l}\text { E. coli QFR bound with } \\
\text { HQNO (Iverson et al., 2002) }\end{array}$ \\
\hline
\end{tabular}

those Q-site inhibitors have different inhibitory specificities on SQR or QFR, e.g. $\alpha$-thenoyltrifluoroacetone (TTFA) and carboxin $(C B X)$ that are vital for studying mitochondrial SQR ubiquinone reduction activity only weakly inhibit bacterial QFRs (Ramsay et al., 1981; Yankovskaya et al., 1996; Maklashina and Cecchini, 1999; Sun et al., 2005;
Huang et al., 2006). According to those specificities, the Q-site inhibitors could be divided into three classes (Fig. 1). Class I inhibitor such as 2-heptyl-4-hydroxyquinoline-N-oxide (HQNO) is the potent inhibitor of QFR, but not effective on SQR activity (Iverson et al., 1999; Maklashina and Cecchini, 1999; Iverson et al., 2002; Miyadera et al., 2003; Maklashina<smiles>CC(C)=CCC1=C(C)C(=O)c2ccccc2C1=O</smiles>

Menaquinone-1<smiles>COC1=C(OC)C(=O)C(CC=C(C)C)=C(C)C1=O</smiles>

Ubiquinone-1<smiles>CCCCCCCc1cc(O)c2ccccc2[n+]1[O-]</smiles>

Figure 1. Classification of known QFR and SQR inhibitors. Menaquinone-1/ubiquinone-1, the substrate of $Q F R / S Q R$, is shown on the top row with their formulas. Their analogues, the inhibitors of QFR or SQR, are shown on the second and third rows and classified. Class I analogues could only inhibit the activity of QFR and class II could only inhibit the activity of SQR, while class III could significantly inhibit both. Each compound's name is depicted under its formula. 
et al., 2006). Class II inhibitors such as CBX, TTFA and atpenins inhibit both $E$. coli and mitochondrial SQR but not QFR (Ramsay et al., 1981; Maklashina and Cecchini, 1999; Matsson and Hederstedt, 2001; Miyadera et al., 2003; Sun et al., 2005; Horsefield et al., 2006; Huang et al., 2006; Ruprecht et al., 2009). In addition, compared to TTFA and CBX, atpenins were reported as more specific and potent inhibitors of SQR (Miyadera et al., 2003; Horsefield et al., 2006). Class III inhibitors were found to be potent inhibitors of both SQR and QFR, including pentachlorophenol (PCP) (Maklashina and Cecchini, 1999; Ruprecht et al., 2009), thiabendazole [2-(4\%-thiazolyl) benzimidazole] (TBZ) (John and Denis, 1981; Armson et al., 1993, 1995; Mileni et al., 2006) and a series of 2-alkyl-4,6-dinitrophenols (Iverson et al., 1999; Maklashina and Cecchini, 1999; Iverson et al., 2002; Yankovskaya et al., 2003).

There are lots of chemical compounds used as the insecticide, fungicide or disinfectant reagents in our current life. However, some of them are still susceptible of their negative effects on human health. Based on our previous purification and structural study of porcine mitochondrial respiratory complex II (in the followings, we call it mitochondrial SQR) (Sun et al., 2005; Huo et al., 2007), five disinfectant compounds, CBX, TBZ, PCP, morantel citrate (MRT) and oxantel pamoate (OXL) (Fig. S1), were selected and investigated on their potential inhibitory effects. $C B X$ was a systemic fungicide and used for probing the Q-site of mitochondrial Complex II (Ramsay et al., 1981; Matsson and Hederstedt, 2001). MRT and OXL (Fig. S1) were used as broad-spectrum anthelmintics in various animal species. TBZ, a kind of benzimidazole with broad anthelmintic activity, is effective in the treatment of various nematode infections in humans and animals (Brown et al., 1961; Cuckler, 1961; Ames et al., 1963; Portugal et al., 2002; Igual-Adell et al., 2004) and shows highly effective against a variety of fungi (Robinson et al., 1978). Pentachlorophenol is an organochlorine compound that was first produced in the 1930s and used as a herbicide, insecticide, fungicide, algaecide, disinfectant and as an ingredient in antifouling paint, was found to be a potent inhibitor of E. coli SQR and QFR (Maklashina and Cecchini, 1999; Ruprecht et al., 2009) and bovine SQR (Afanas'eva and Kostyrko, 1986).

In this study, by measuring the inhibitory effect on ubiquinone reduction activity, we found, more clearly than before, besides PCP and CBX, TBZ is also an effective inhibitor of the ubiquinone reduction of mitochondrial SQR with an $I_{50}$ value of $\sim 100 \mu \mathrm{mol} / \mathrm{L}$. Crystallographic studies showed that both PCP and TBZ could bind exclusively to the $Q$-site. Interestingly, by comparing with other known inhibitors of SQR, a special water molecule mediated inhibitor-binding feature is revealed from the TBZ bound structure. Both measured $I_{50}$ values and calculated binding affinity constants indicated that TBZ exhibits more inhibitory effects on porcine SQR than its nematode equivalent, which raises the dose safety concern for TBZ usage during nematode infections treatment and fruit storage.

\section{RESULTS AND DISCUSSION}

\section{Comparison of the inhibitory effects of different disinfectant compounds on mitochondrial SQR ubiquinone reduction}

Originally, we obtained several disinfectant compounds from pharmaceutical companies and sought to check their negative effects on several important metabolic enzymes in human. We selected the mitochondrial $S Q R$ as a target, which plays a very important role in citric cycle and oxidativephosphorylation process of energy metabolism. Any disinfectant compound that could severely inhibit the enzyme activity of mitochondrial SQR is worthy of attention for its usage in agriculture and food industry.

Compared with the previous purification procedure (Sun et al., 2005; Huo et al., 2007), the further chromatography purification by CHT-1 and Superdex 200 column enabled us to obtain the porcine heart mitochondrial SQR with a purity up to $95 \%$ (Fig. 2A), thus minimizing the potential influences from contaminations in enzymatic activity assay experiments.

Based on the measurements of the ubiquinone reduction activity with different compounds and under varied concentrations (Fig. 2B), it was found that among those five disinfectant compounds MRT and OXL had no inhibitory effects on the mitochondrial SQR activity, while CBX, PCP and TBZ could greatly inhibit the ubiquinone reduction. CBX has already been reported as a specific class II inhibitor of mitochondrial SQR (Maklashina and Cecchini, 1999; Huang et al., 2006) and PCP was also found to be able to inhibit both the activity of SQR and QFR (Maklashina and Cecchini, 1999; Ruprecht et al., 2009). Furthermore, our data revealed that $\mathrm{PCP}$ had more inhibitory effects than $\mathrm{CBX}$ with an $\mathrm{IC}_{50}$ value $\sim 50 \mu \mathrm{mol} / \mathrm{L}$. TBZ could inhibit the activity of mitochondrial SQR significantly even at low concentrations. And its inhibitory effect was much stronger than CBX although they had a similar $I C_{50}$ value of $\sim 100 \mu \mathrm{mol} / \mathrm{L}$.

TBZ has been reported to be able to kill a variety of fungi and nematodes by potentially inhibiting their succinate dehydrogenase-fumarate reductase system (Allen and Gottlieb, 1970). In this study, TBZ was found, more evidently, to be an efficient inhibitor of mitochondrial SQR. Therefore, we categorize TBZ as class III inhibitors (Fig. 1).

\section{A water molecule mediated inhibitor-binding feature defined from the structure of mitochondrial SQR bound with TBZ}

After the inhibitory assay, crystallographic approach was further applied to study the detailed interactions between the disinfectant compound (CBX, PCP or TBZ) and mitochondrial 
A

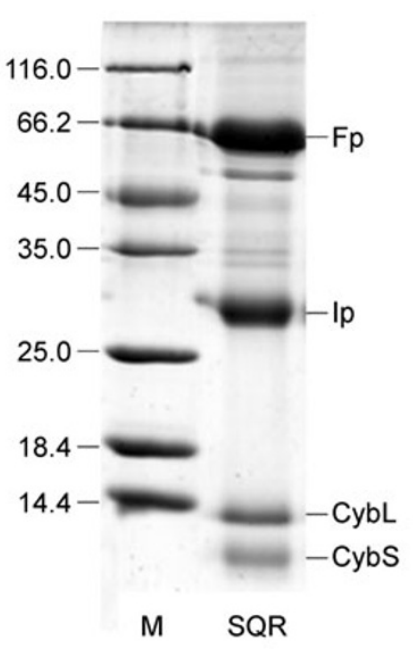

B

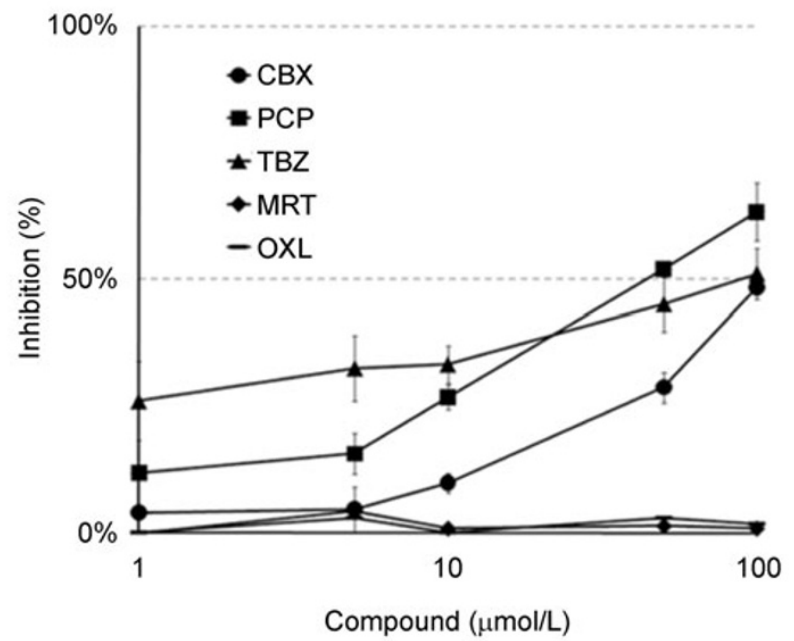

Figure 2. Purification of porcine heart mitochondrial SQR and its ubiquinone reduction inhibition by disinfectant compounds. (A) SDS-PAGE of purified mitochondrial SQR from porcine heart. Four subunits (flavoprotein, Fp, $68 \mathrm{kDa}$; iron-sulfur protein, Ip, $29 \mathrm{kDa}$; cytochrome b binding proteins, CybL, $15 \mathrm{kDa}$ and CybS, $11 \mathrm{kDa}$ ) could be observed clearly with equal stoichiometry. (B) Inhibitory effects on the mitochondrial SQR ubiquinone reduction activity by five disinfectant compounds. See MATERIALS AND METHODS for the detailed assay methods. The ubiquinone reduction activity of mitochondrial SQR without adding disinfectant compounds was taken as the reference activity and the inhibition level of compound ( $y$-axis) was calculated as (1 - measured activity/reference activity). The X-axis represents the compound concentration with log-scale. Error bars indicate S.E.M. $(n=3)$.

SQR. Because the crystal structures of $C B X$ bound to either bacterial SQR (Ruprecht et al., 2009) or mitochondrial SQR (Huang et al., 2006) have been solved, we co-crystallized the porcine heart mitochondrial SQR with PCP or TBZ and solved their complex structures. The structure of SQR-PCP was finally refined to $2.6 \AA$ with $R_{\text {work }} 24.2 \%$ and $R_{\text {free }} 28.6 \%$ and that of SQR-TBZ to $2.8 \AA$ with $R_{\text {work }} 21.4 \%$ and $R_{\text {free }} 27.0 \%$ (Table 2). The position and orientation of PCP and TBZ were clearly assigned according to the $2 \mathrm{FoFc}$ map around the $\mathrm{Q}$ site (Fig. 3A and 3B).

Similar to the ubiquinone binding, residues Tyr-D91 and Trp-B173 play important roles to interact with PCP via ubiquitous hydrogen bonds (Fig. $3 \mathrm{~A}$ and Table 3 ). Besides hydrogen bonds, residues Met-C39, Pro-B169 and Ile-B218 coordinate PCP via the hydrophobic interactions (Table 3). Comparison between mitochondrial SQR-PCP complex structure and E. coli SQR-PCP complex structure (PDB 2WDR) (Fig. S2 and Table 3) reveals a rather similar binding mode and further confirms the conservation of SQR ubiquinone-binding pocket from bacteria to mammalian.

Surprisingly, both the binding position of TBZ at the Q-site and its interaction feature with the mitochondrial SQR exhibit significant differences with either the ubiquinone (Fig. 3B) or other inhibitors' binding (Fig. 3C, 3D and S3).

Without the significant changes of the whole SQR structure, TBZ is shifted away from the original Q-site that is defined by ubiquinone or PCP binding and becomes closer to the helix $2 \mathrm{~L}$ of the large cytochrome $b$ binding protein $(\mathrm{CybL})$ and deeper into the binding pocket (Fig. 3B). Although TryD91 and Trp-B173 are still playing the roles of TBZ recognition and binding, their hydrogen binding interactions with the nitrogen of TBZ benzimidazole group are actually mediated by a water molecule (W1). The temperature factor of the water molecule (W1) is $34.4 \AA^{2}$, which is lower than the overall average value $56.5 \AA^{2}$, suggesting this water molecule is stable and significant for the TBZ binding. Furthermore, another hydrogen bond was found between the second nitrogen of TBZ benzimidazole group and the hydroxyl oxygen of Ser-C42. Besides the TBZ benzimidazole group, TBZ also uses its thiazole group to interact with the mitochondrial SQR. The hydrogen bond between the thiazole sulfur and the guanidyl group of Arg-C46 was clearly confined by the strong electron density.

Compared with the major (CBX) or specific (atpenin A5) inhibitor of mitochondrial SQR (Fig. 3C and 3D), TBZ is closer to the helix 2L of CybL but away from Try-D91 and Trp-B173, as a result that there is a water molecule (W1) existing to mediate the conserved hydrogen bond interaction between TBZ and those two residues. The hydrogen bond between Ser-C42 and TBZ could also be observed in the CBX or atpenin A5 bound structures (Table 3 ), revealing the importance of Ser-C42 for inhibitor binding specificity. The hydrogen bond formed between Arg-C46 and TBZ has never been observed from any other inhibitors and endows a 
Table 2 Statistics of the data collection and structure refinement

\begin{tabular}{|c|c|c|}
\hline & SQR-TBZ complex & SQR-PCP complex \\
\hline \multicolumn{3}{|c|}{ Data collection and processing ${ }^{a}$} \\
\hline Space group & $\mathrm{P} 2{ }_{1}{ }_{1} 2_{1}$ & $\mathrm{P} 2{ }_{1} 2_{1} 2_{1}$ \\
\hline Unit cell $(\AA)$ & $a=70.7, b=83.4, c=294.0$ & $a=70.3, b=83.4, c=293.1$ \\
\hline Wavelength $(\AA)$ & 1.0000 & 1.0000 \\
\hline Resolution limit $(\AA)$ & $50.0-2.8(2.87-2.80)$ & $50.0-2.6(2.69-2.60)$ \\
\hline Total reflections & 74569 (3976) & $93777(6226)$ \\
\hline Unique reflections & $41586(2322)$ & $50419(3557)$ \\
\hline Completeness (\%) & $94.6(81.2)$ & $93.9(67.8)$ \\
\hline $\mathrm{R}_{\text {merge }}(\%)^{\mathrm{b}}$ & $10.0(42.7)$ & $13.6(47.6)$ \\
\hline$<\mathrm{I} / \sigma(\mathrm{I})>$ & $12(1.7)$ & $20.0(1.7)$ \\
\hline \multicolumn{3}{|c|}{ Refinement } \\
\hline Resolution range $(\AA)$ & $50.0-2.8$ & $50.0-2.6$ \\
\hline $\mathrm{R}_{\text {work }}(\%)^{\mathrm{c}}$ & 21.4 & 24.2 \\
\hline $\mathrm{R}_{\text {free }}(\%)^{\mathrm{c}}$ & 27.0 & 28.6 \\
\hline r.m.s.d. of bonds $(\AA)^{d}$ & 0.007 & 0.007 \\
\hline r.m.s.d. of angle $\left({ }^{\circ}\right)^{d}$ & 1.243 & 1.237 \\
\hline Average B-factor $\left(\AA^{2}\right)$ & 56.5 & 60.0 \\
\hline
\end{tabular}

potential new recognition site for designing new specific inhibitors. Besides CBX and atpenin A5, TTFA and DNP-17 were also reported to be the inhibitors of SQR (Ramsay et al., 1981; Maklashina and Cecchini, 1999) and their structures bound to SQR were reported previously (Yankovskaya et al., 2003; Sun et al., 2005). Compared with those inhibitors, TBZ exhibits significantly different binding motif again (Fig. S3). Taking the numbers of hydrogen bonds and hydrophobic contacts into account, TBZ has many contact sites with the mitochondrial SQR, more than PCP, TTFA and DNP-17, similar to $\mathrm{CBX}$ and atpenin A5 although the water molecules play important roles for TBZ binding (Table 3 ). It is exactly worthy to compare the inhibitory activity between TBZ and atpenin A5; however we did not have atpenin A5 compound available at hand. In conclusion, the comparison with current known SQR inhibitors demonstrates that TBZ could inhibit the activity of mitochondrial SQR via a special water-mediated binding feature that was never reported before for other known inhibitors.

\section{Porcine SQR is more sensitive to the inhibition by TBZ than nematode QFR}

Thiabendazole is used as a broad spectrum anthelmintic in various animal species and is also employed for the control of parasitic infection in humans. The quinol:fumarate reductase (QFR) in the nematodes has been discussed and considered as a possible target after TBZ treatment (Allen and Gottlieb,
1970; John and Denis, 1981; Armson et al., 1993, 1995). It should be noted that, unlike bacterial QFR (Iverson et al., 1999), nematode QFR, e.g. A. suum QFR, has an SQR-like primary structure. Its four subunits, flavoprotein, iron-sulfur binding protein, large cytochromce $b$ binding protein and small cytochrome $\mathrm{b}$ binding protein, all exhibit more similarities of $83 \%, 81 \%, 60 \%$ and $58 \%$, respectively, with the bacterial or mitochondrial SQR than the bacterial QFR (Kuramochi et al., 1994; Amino et al., 2000, 2003) (Fig. S4). And the residues around the succinate/fumarate binding site as well as the quinone/quinol binding site are similar to the mammalian mitochondrial SQR (Fig. 4). So it is interesting but not surprising that the QFR from $A$. suum showed the high SQR activity in vitro (Kita et al., 2002; Iwata et al., 2008). However, due to the large similarity between the nematodes QFR and the mammalian mitochondrial SQR, the concern about the safety of using TBZ as an efficient anthelmintic should be raised. It was reported that the $\mathrm{IC}_{50}$ value of $T B Z$ on the inhibition of Strongyloides ratti QFR activity in the mitochondrion was $0.46 \mathrm{mmol} / \mathrm{L}$ (Armson et al., 1995) whereas its measured $I C_{50}$ value on the inhibition of the purified porcine mitochondrial SQR activity was $0.1 \mathrm{mmol} / \mathrm{L}$ in this work. The smaller $I_{50}$ value indicates porcine $S Q R$ is more sensitive to the inhibition by TBZ than nematode QFR.

To further compare the inhibition potencies of TBZ between porcine and nematode, we constructed the $3 D$ structural model of $A$. suum QFR based on the porcine SQR crystal structure by MODELLER (Eswar et al., 2006). Comparison of 

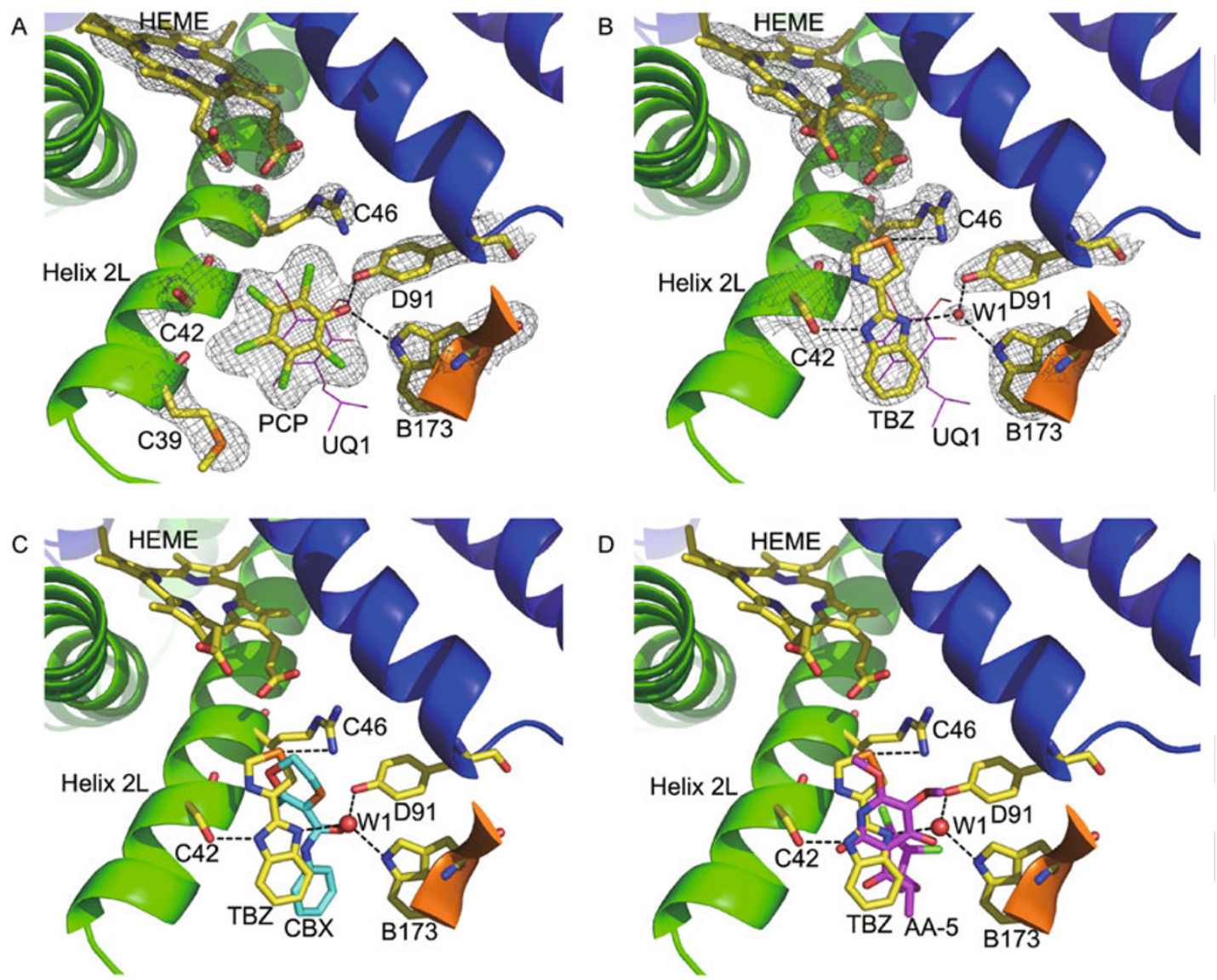

Figure 3. PCP and TBZ bound mitochondrial SQR structures at the Q-site and comparisons with other inhibitors. (A and B) SQR-PCP (A) and SQR-TBZ (B) structures at the Q-site. (C and D) Comparison of SQR inhibitor binding motifs between TBZ and carboxin (PDB 2FBW) in (C) or atpenin A5 (PDB 3AEE) in (D). PCP, TBZ, heme and the important residues at the Q-site are labeled and shown as the stick representation with the carbon atom colored by yellow, oxygen by red, nitrogen by blue and chloride by green and sulfur by orange. Carboxin and atpenin A5 are colored by cyan and magenta respectively. Water molecules are shown in CPK representation. The $2 \mathrm{Fo}-\mathrm{Fc}$ electron density map is shown as grey mesh and contoured at $1.5 \sigma$ in $(A)$ and $1.2 \sigma$ in (B). SQR substrate, ubiquinone-1, is shown as the purple line representation at the relevant position according to the previous crystal structure of porcine SQR (PDB 1ZOY) (Sun et al., 2005). The iron-sulfur protein is depicted as orange cartoon, CybL as green with the second helix labeled (Helix $2 \mathrm{~L}$ ) and CybS as blue. The hydrogen bonds are shown as black dotted lines. All the figures in this paper are generated by PyMOL (DeLano, 2002, http://www.pymol.org) and polished in Photoshop (Adobe, Inc.).

the A. suum QFR model with the porcine SQR-TBZ structure revealed the similar environment around the TBZ binding pocket and the conserved specific residues (Tyr-D91, TrpB173, Ser-C42 and Arg-C46) for inhibitor binding (Fig. 4A). However, the residues surrounding the binding pocket at the helix $2 \mathrm{~L}$ of CybL are different between porcine SQR and $A$. suum QFR. In the porcine SQR structure, there are Trp-C35, Met-C39 and Ile-C43 along the CybL helix $2 \mathrm{~L}$, while there are Pro-C65, Trp-C69 and Gly-C73 at the equivalent positions in the A. suum QFR model (Fig. 4A). The substitutions from TrpC35/lle-C43 to Pro-C65/Gly-C73 around the entry of the pocket resulted in a more open entrance of the pocket (Fig. 4B and 4C), which might be responsible for the different binding strength of TBZ. Furthermore, the substitution of middle size residue Met-C39 with a large residue Trp-C69 may yield a smaller binding pocket and the residue Trp-C69 might prohibit TBZ binding closer to the CybL helix $2 \mathrm{~L}$, which might negatively influence the TBZ binding (Fig. 4B and 4C). In addition, we found the three substituted residues (Pro-C65, Gly-C73 and Trp-C69) are highly conserved among the nematodes QFR/SQRs (Fig. 4D and S5), suggesting their ubiquitous roles in the binding with TBZ. The differences of the residues at the CybL helix $2 \mathrm{~L}$ between porcine $S Q R$ and A. suum QFR might be responsible for the different inhibitory effects of TBZ on these two species.

The binding affinities of TBZ to porcine SQR and $A$. suum QFR are further calculated and compared by computational approach. The binding free energies and the inhibition constants of PCP or TBZ bound to porcine SQR or A. suum QFR were computed and evaluated by molecular docking 
Table 3 Hydrogen bonds and hydrophobic interactions of binding site ${ }^{\text {a }}$

\begin{tabular}{|c|c|c|c|c|c|c|c|c|c|c|c|c|c|c|}
\hline \multirow{3}{*}{$\begin{array}{l}\text { Substrate } \\
\text { /inhibitor }\end{array}$} & \multirow[t]{3}{*}{ Protein } & \multirow{3}{*}{$\begin{array}{l}\text { PDB } \\
\text { code }\end{array}$} & \multirow{3}{*}{$\begin{array}{c}\text { Porcine } \\
\text { Avian } \\
\text { E. coli }{ }^{\text {b }}\end{array}$} & \multirow{2}{*}{$\begin{array}{l}\text { B173W } \\
\text { B173W }\end{array}$} & \multirow{2}{*}{$\begin{array}{l}\text { D91Y } \\
\text { D58Y }\end{array}$} & \multirow{2}{*}{$\begin{array}{l}\text { B169P } \\
\text { B169P }\end{array}$} & \multirow{2}{*}{$\begin{array}{l}\text { B218I } \\
\text { B218I }\end{array}$} & \multirow{2}{*}{$\begin{array}{l}\text { C30I } \\
\text { C27I }\end{array}$} & \multirow{2}{*}{$\begin{array}{l}\text { C35W } \\
\text { C32W }\end{array}$} & \multirow{2}{*}{$\begin{array}{l}\text { C43I } \\
\text { C40I }\end{array}$} & \multirow{2}{*}{$\begin{array}{l}\text { C39M } \\
\text { C36M }\end{array}$} & \multirow{2}{*}{$\begin{array}{l}\text { C42S } \\
\text { C39S }\end{array}$} & \multirow{3}{*}{$\begin{array}{l}\text { B216H } \\
\text { B216H } \\
\text { B207H }\end{array}$} & \multirow{3}{*}{$\begin{array}{l}\text { C46R } \\
\text { C43R } \\
\text { C31R }\end{array}$} \\
\hline & & & & & & & & & & & & & & \\
\hline & & & & $\mathrm{B} 164 \mathrm{~W}^{\mathrm{b}}$ & D83Y & B160P & B209I & C15L & $\mathrm{C} 20 \mathrm{~F}$ & C28I & C24A & C27S & & \\
\hline \multirow[t]{2}{*}{$\begin{array}{l}\text { Ubiqui- } \\
\text { none }\end{array}$} & $\begin{array}{l}\text { E.coli } \\
\text { SQR }\end{array}$ & 1NEK & & $\mathrm{hb}$ & $\mathrm{hb}$ & + & + & + & + & + & & & & \\
\hline & $\begin{array}{c}\text { Porcine } \\
\text { SQR }\end{array}$ & $1 Z O Y$ & & $\mathrm{hb}$ & $\mathrm{hb}$ & N.A. & N.A. & + & + & N.A. & & & & + \\
\hline DNP-17 & $\begin{array}{l}\text { E.coli } \\
\text { SQR }\end{array}$ & $1 \mathrm{NEN}$ & & $\mathrm{hb}$ & $\mathrm{hb}$ & + & + & + & & + & & & & \\
\hline TTFA & $\begin{array}{c}\text { Porcine } \\
\text { SQR }\end{array}$ & $1 \mathrm{ZP0}$ & & $\mathrm{hb}$ & $\mathrm{hb}$ & + & & & & & & $\mathrm{hb}$ & + & + \\
\hline \multirow[t]{2}{*}{ PCP } & $\begin{array}{l}\text { E.coli } \\
\text { SQR }\end{array}$ & $2 \mathrm{WDR}$ & & $\mathrm{hb}$ & $\mathrm{hb}$ & + & & & & + & & & & \\
\hline & $\begin{array}{c}\text { Porcine } \\
\text { SQR }\end{array}$ & 3SFD & & $\mathrm{hb}$ & $\mathrm{hb}$ & + & + & & & & + & & & \\
\hline \multirow[t]{2}{*}{ CBX } & $\begin{array}{l}\text { E.coli } \\
\text { SQR }\end{array}$ & $2 W D Q$ & & $\mathrm{hb}$ & $\mathrm{hb}$ & + & + & + & + & + & & + & + & + \\
\hline & $\begin{array}{l}\text { Avian } \\
\text { SQR }\end{array}$ & $2 \mathrm{FBW}$ & & $\mathrm{hb}$ & $\mathrm{hb}$ & + & + & + & + & + & + & $\mathrm{hb}$ & + & + \\
\hline \multirow[t]{2}{*}{ AA5 } & $\begin{array}{l}\text { E.coli } \\
\text { SQR }\end{array}$ & $2 A C Z$ & & $\mathrm{hb}$ & $\mathrm{hb}$ & + & + & + & + & + & & $\mathrm{hb}$ & $\mathrm{hb}$ & + \\
\hline & $\begin{array}{c}\text { Porcine } \\
\text { SQR }\end{array}$ & 3AEE & & $\mathrm{hb}$ & $\mathrm{hb}$ & + & + & + & & + & & & + & \\
\hline TBZ & $\begin{array}{c}\text { Porcine } \\
\text { SQR }\end{array}$ & 3SFE & & W & W & + & + & & + & + & + & $\mathrm{hb}$ & + & $\mathrm{hb}$ \\
\hline
\end{tabular}

${ }^{\mathrm{a}} \mathrm{Hb}$, hydrogen bonds; +, hydrophobic contacts; $\mathrm{W}$, water mediated interaction.

${ }^{\mathrm{b}}$ The corresponding residues for compound binding are given as $<$ chain name $><$ residue number $><$ residue name $>$ from three different species (porcine, avian and E.coli) in the respective rows.

program AutoDock 4.2 (Morris et al., 2009). As a result (Fig. $4 \mathrm{E}$ ), the calculated constants for PCP and TBZ binding to the porcine SQR were $\sim 48 \mu \mathrm{mol} / \mathrm{L}$ and $\sim 94 \mu \mathrm{mol} / \mathrm{L}$, which were in accordance with their measured $I_{50}$ values upon the porcine SQR activity (Fig. 2B). And the weaker binding affinity of TBZ to the $A$. suum QFR is confirmed again by the larger calculated binding constant $\sim 263 \mu \mathrm{mol} / \mathrm{L}$, which is in agreement with the previously reported $I_{50}$ value $460 \mu \mathrm{mol} / \mathrm{L}$ for TBZ inhibition to the Strongyloides ratti QFR (Armson et al., 1995).

\section{Implications from studying TBZ inhibition of mitochondrial SQR}

TBZ has been registered in many countries as a parasiticide and fungicide. As an antiparasitic, it has broad anthelmintic activity and is used to control roundworms (such as Strongyloides ratti), hookworms, and other helminth species which attack wild animals, livestock and humans (Brown et al., 1961; Cuckler, 1961; Ames et al., 1963; Portugal et al., 2002; Igual-Adell et al., 2004). The SDH/QFR system in nematodes has received more attention as a potential target of TBZ (Allen and Gottlieb, 1970) although John and Denis
(1981) suggested that the SDH/QFR complex is not the primary chemotherapeutic attack target of TBZ in Aspiculuris tetraptera and Ascaris suum. Previous works have shown that TBZ has significant inhibitory effects on QFR than SDH in nematodes from $A$. tetraptera, A. suum (John and Denis, 1981) and Strongyloides ratti (Armson et al., 1993, 1995). TBZ was also shown to be highly effective against a variety of fungi (Robinson et al., 1965, 1978). In most cases of the postharvest application, TBZ is now widely used as a food additive (post-harvest fungicide) in order to prevent fruits (e.g. oranges and bananas) and vegetables from deteriorating during storage and transportation.

However the toxic effects of TBZ were frequently observed in humans (Pawlowski and Skrzypinska, 1972; Golden et al., 1974; Rex et al., 1983; Manivel et al., 1987; Roy et al., 1989; Bion et al., 1995) and animals (Robinson et al., 1965; Ogata et al., 1984; Tada et al., 2001); the toxicity mechanism of TBZ on mammalian system has not been understood. Our study in this work demonstrated that TBZ is a potent inhibitor of porcine mitochondrial SQR with an $\mathrm{IC}_{50}$ value $\sim 100 \mu \mathrm{mol} / \mathrm{L}$ and the interaction motif of TBZ bound to mitochondrial SQR is surprisingly different from all current known SQR inhibitors. The number of hydrogen bonds and hydrophobic contacts of $T B Z$ to $S Q R$ is similar to the SQR specific inhibitor atpenin A5, 
A

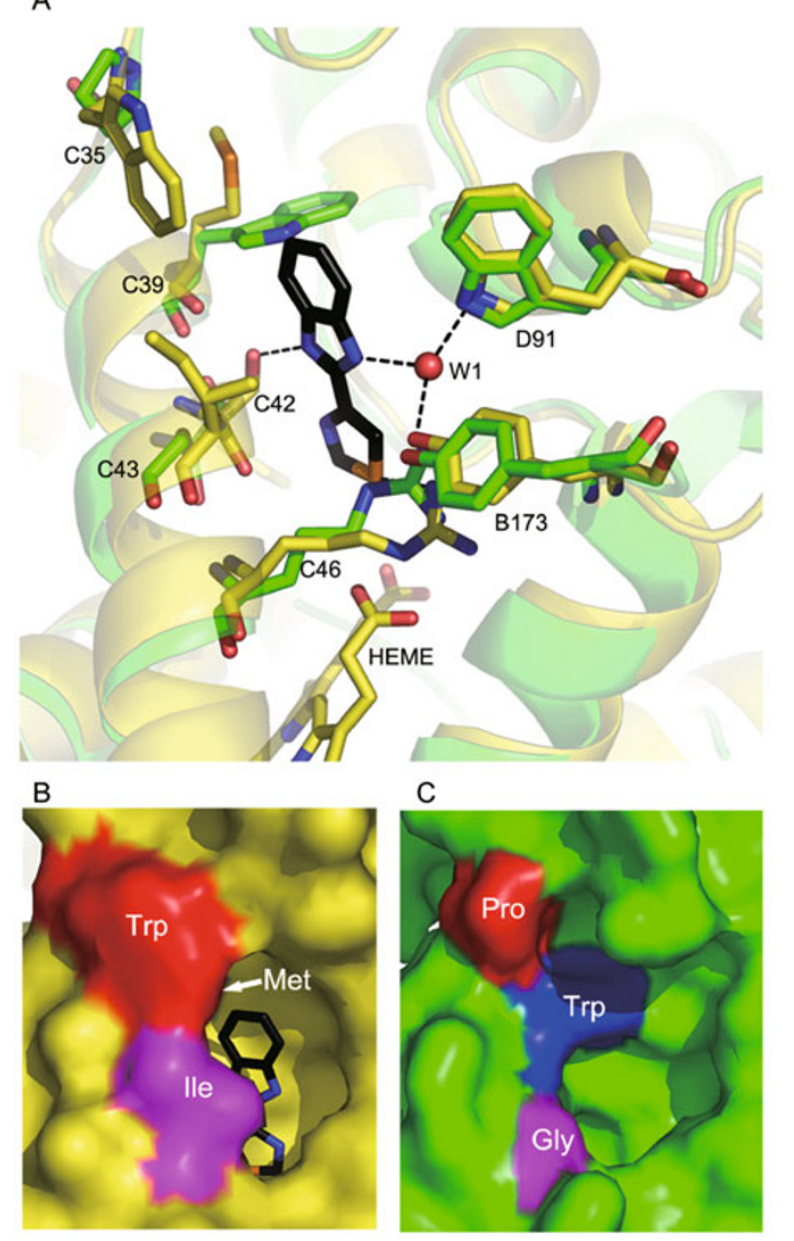

D

Sus_scrofa

Sus_scrofa Homo_sapiens Caenorhabditis_briggsae Brugia_malayi Ascaris_suum

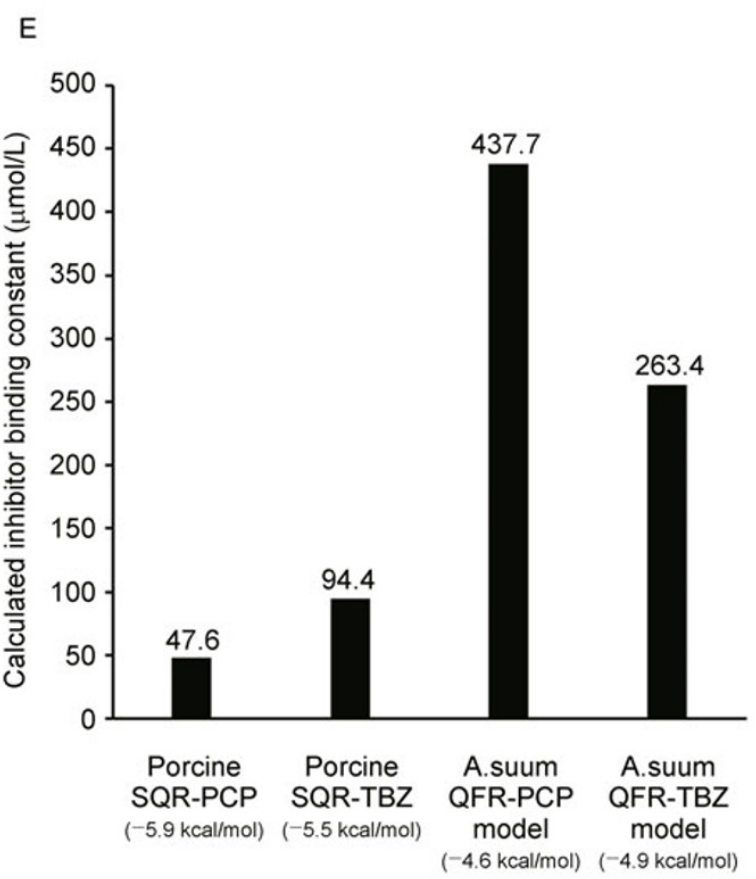

Figure 4. Comparison of Q-sites and inhibitor binding affinity between porcine SQR and $A$. suum QFR. (A) Superposition of porcine SQR (SQR-TBZ structure) and A. suum QFR (modeling) at the Q-site. Porcine SQR and A. suum QFR are shown in yellow and green, respectively. The conserved residues Trp-D91 and Tyr-B173 and the residues on the Helix 2L are shown as the stick model and labeled respectively. TBZ from porcine SQR-TBZ structure is shown in black stick. The color scheme for each atom (except carbon atom) is the same with Fig. 3. (B and C) Surface presentations of the $Q$-site pockets of porcine SQR with TBZ binding (B) and A. suum QFR model (C). The regions of Trp-C35 (B)/Pro-C65 (C), Met-C39 (B)/Trp-C69 (C) and Ile-C43 (B)/Gly-C73 (C) are mapped onto the surfaces, labeled and colored by red, blue and pink, respectively. TBZ from porcine SQR-TBZ structure is shown in black stick. (D) Sequence alignment of Helix $2 \mathrm{~L}$ among Sus scrofa, Homo sapiens, Gallus gallus domesticus, Brugia malayi, Ascaris suum, Caenorhabditis elegans and Caenorhabditis briggsae. The identical residues are colored by white and highlighted by red and those with a similarity larger than $70 \%$ colored by red and highlighted by white. And the different key residues between vertebrate and nematode are marked by the black triangles. The secondary structural elements come from the crystal structure of Sus scrofa SQR (1ZOY). The conserved residues (Ser-C42 and Arg-C46) for TBZ specific binding are marked by the black circles. The alignment was generated by ClustalW 1.7 (Thompson et al., 1994) and colored by ESPript (Franklin and Gosling, 1953; Gouet et al., 1999). (E) The calculated binding free energies and the inhibitor binding constants of PCP or TBZ bound to porcine SQR or $A$. suum QFR. The calculated inhibitor binding constants are shown as histogram and the calculated binding free energies are shown in the brackets accordingly.

indicating TBZ would be a more specific inhibitor compared with others like TTFA, PCP, etc.

Due to the significant toxic effect of TBZ on the ubiquinone reduction activity of mitochondrial SQR, the dose of using TBZ as a drug or food additives must be carefully controlled. In fact, the use of thiabendazole was experimentally proved with a relatively high tolerance level; the acceptable daily intake (ADI) for human is $0-0.1 \mathrm{mg} / \mathrm{kg}$ body weight (Joint FAO/WHO Meeting, 2002). Since our work here further confirmed the toxicity of TBZ to the mammalian respiratory systems, more cautions should be given for the long-term application of TBZ. 


\section{MATERIALS AND METHODS}

\section{Preparation of mitochondrial SQR from porcine heart}

The porcine heart mitochondrial SQR was purified as previously described for crystallization study (Sun et al., 2005; Huo et al., 2007). In order to obtain a high purity for enzymatic study, the crude sample after ammonium sulfate precipitation was further purified by ceramic hydroxyapatite (CHT) and gel filtration chromatography. In brief, the fraction from ammonium sulfate precipitation at 35\%-45\% saturation was dissolved in buffer A containing $50 \mathrm{mmol} / \mathrm{L}$ potassium phosphate, $\mathrm{pH} 7.2$ and $0.5 \%$ Thesit (Sigma) to a final concentration of $5 \mathrm{mg} / \mathrm{mL}$ and then applied to the CHT-1 column (Bio-Rad, USA) that was equilibrated with buffer $A$ using BioLogic Duoflow system (Bio-Rad, USA). After the crude SQR was loaded, the column was eluted with buffer $A$ for $20 \mathrm{~min}$ at a flow rate of $1 \mathrm{~mL} / \mathrm{min}$ and the mitochondrial SQR was collected from the flow-through fraction before the CHT-1 column was washed with $0.5 \mathrm{~mol} / \mathrm{L}$ potassium phosphate buffer $(\mathrm{pH}$ 7.2) containing $0.5 \%$ Thesit. The collected SQR fractions were concentrated by ultra-filtration using Amicon Ultra-4 centrifugal filters (MWCO $50 \mathrm{kDa}$, Millipore) and then applied to Superdex $200 \mathrm{HR}$ 10/ 300GL (GE Healthcare) with buffer B containing $25 \mathrm{mmol} / \mathrm{L}$ HEPES, $\mathrm{pH} 7.2,150 \mathrm{mmol} / \mathrm{L} \mathrm{NaCl}, 200 \mathrm{mmol} / \mathrm{L}$ sucrose, $0.45 \%$ sodium cholate and $1 \mathrm{mmol} / \mathrm{L}$ EDTA. The final eluted fraction by gel-filtration contained the highly purified mitochondrial SQR that was used for further enzymatic study.

In this work, the protein concentrations were determined by BCA (bicinchoninic acid) method (Stoscheck, 1990) with bovine serum albumin as a standard and SDS-PAGE was performed using $15 \%$ polyacrylamide gel.

\section{Enzymatic activity assay}

The ubiquinone reduction activity of porcine mitochondrial SQR was measured by monitoring the optical absorbance change at $600 \mathrm{~nm}$ in the presence of 2,3-dimethoxy-5-methyl-6-geranyl-1,4-benzoquinone (UQ2) and 2,6-dichlorophenolindophenol (DCIP) that was used as a terminal electron acceptor (Kita et al., 1989). The final assay mixture contained $50 \mathrm{mmol} / \mathrm{L}$ sodium phosphate, $\mathrm{pH} 7.4,100 \mu \mathrm{mol} / \mathrm{L}$ UQ2, $100 \mu \mathrm{mol} / \mathrm{L}$ DCIP, $10 \mathrm{mmol} / \mathrm{L}$ sodium succinate and $1 \mathrm{mmol} / \mathrm{L}$ EDTA. The optical absorbance at $600 \mathrm{~nm}$ was decreased after adding porcine mitochondrial SQR $(1 \mu \mathrm{g})$ and the SQR activity was calculated by using the extinction coefficient of DCIP $\left(21 \mathrm{mmol} \cdot \mathrm{L}^{-1} \cdot \mathrm{cm}^{-1}\right.$ at $\left.600 \mathrm{~nm}\right)$. The inhibitory effects on SQR activity by five disinfectant compounds were measured at three kinds of compounds' concentrations of 1,10 and $100 \mu \mathrm{mol} / \mathrm{L}$. Each compound was dissolved in $100 \%$ dimethyl sulfoxide (DMSO) to a final concentration of $10 \mathrm{mmol} / \mathrm{L}$ as a stock solution and then diluted as needed. In each assay, the compound was added to the assay mixture prior to adding porcine mitochondrial SQR. All the enzymatic assay experiments were performed at $25^{\circ} \mathrm{C}$ by using Hitachi U2010 spectrophotometer and repeated three times.

\section{Co-crystallization, data collection and structure determination}

Co-crystallization between porcine mitochondrial SQR and the inhibitor (PCP or TBZ) was performed with the condition as previously described (Sun et al., 2005; Huo et al., 2007). In brief, the SQR harvested from ammonium sulfate precipitation at $40 \%-43.5 \%$ saturation was dissolved into the buffer containing $20 \mathrm{mmol} / \mathrm{L}$
HEPES, pH 7.2, $200 \mathrm{mmol} / \mathrm{L}$ sucrose, $100 \mathrm{mmol} / \mathrm{L} \mathrm{NaCl}$ and $0.5 \mathrm{mmol} / \mathrm{L}$ EDTA. The ubiquinone-reduction inhibitor (PCP or TBZ) along with the succinate dehydrogenation inhibitor oxaloacetate (OAA) was added with a final concentration of $2 \mathrm{mmol} / \mathrm{L}$ into the buffered SQR $(25 \mathrm{mg} / \mathrm{mL})$ and the mixture was incubated on ice for $1 \mathrm{~h}$ before subjecting to crystallization with the similar condition to the native SQR (Sun et al., 2005; Huo et al., 2007). Crystals appeared after 2-day growth at $16^{\circ} \mathrm{C}$ and gained their good shape and high diffraction quality after further $1-2$ days. The diffraction data sets were collected at $95 \mathrm{~K}$ with the wavelength of $1.0 \AA$ at the beam-line $B L 17 \mathrm{~A}$, Photon Factory, Tsukuba, Japan. Data were processed, integrated, merged and scaled using HKL2000 (Otwinowski and Minor, 1997). The crystals belong to the orthorhombic space group $P 2{ }_{1} 2_{1} 2_{1}$ with similar unit cell to the native SQR (Sun et al., 2005; Huo et al., 2007).

The crystal structures of SQR bound to PCP (SQR-PCP) and bound to TBZ (SQR-TBZ) were solved by molecular replacement using the program PHASER (McCoy et al., 2005) with the coordinates of the native SQR structure (PDB 1ZOY) as the initial model. Using the algorithm (http://www.doe-mbi.ucla.edu/ sawaya/anisoscale) supplied by David Eisenberg's group (Strong et al., 2006), diffraction anisotropic correction was performed to improve the quality of the final electron density map. The iterative model building and refinement was performed by using COOT (Emsley and Cowtan, 2004) and REFMAC (Murshudov et al., 1997). And the stereochemistry of the final structures passed through the validation by PROCHECK (Laskowski et al., 1993). The statistics for data processing and structure refinement are summarized in Table 2.

\section{Modeling, inhibitor docking and binding free energy calculation}

Based on the crystal structures of SQR-PCP and SQR-TBZ, the corresponding inhibitor bound structures of QFR (QFR-PCP and QFR-TBZ) from Ascaris suum (A. suum) were modeled by using MODELLER (Eswar et al., 2006). AUTODOCK 4.2 package (Morris et al., 2009) was used to calculate the binding free energies and inhibition constants of PCP and TBZ to the porcine SQR (based on crystal structures) and nematode QFR (based on molecular modelings), respectively. Firstly, in each round of calculation, the system was pre-treated with AutodockTools by adding polar hydrogens, assigning partial atomic Gasteiger charges and defining the solvation parameters and the rotatable torsions on the protein and the inhibitors. Secondly, the three dimensional grids were generated with Autogrid 4.2. Finally, Autodock 4.2 was used to calculate the binding free energy. The empirical free energy function and Lamarckian generic algorithm were used in the docking with an initial population of 150 randomly placed individual inhibitors and a maximum of 25,000,000 energy evaluations. The docking results were clustered by root mean square deviation (RMSD) and the lowest free energy conformations were selected for the final output. The binding free energies and the inhibition constants of PCP or TBZ bound to porcine SQR or nematode QFR were further evaluated. For each case, the docking results fall into single clusters based on ligand RMSD, suggesting the validity of the docking.

\section{ACCESSION NUMBER}

The crystal structure coordinates of SQR-PCP and SQR-TBZ were deposited in the Protein Data Bank (PDB) with accession numbers 3SFD and 3SFE, respectively. 


\section{ACKNOWLEDGEMENTS}

We acknowledge the Photon Factory, Tsukuba, Japan for the provision of synchrotron radiation facilities and thank Dr. Yusuke Yamada, Dr. Kazutaka Demura and Dr. Takayuki Kubota for their assistances in using beamline BL17A. We would also like to thank Supercomputing Center of Chinese Academy of Sciences (SCCAS) for computantional resources. We are grateful to Kai Zhang (Fei Sun's group) for his help on data processing and Ruigang Su (Fei Sun's group) for his assisstance in lab management. We also thank Xudong Zhao, Ruimin Zheng and Su liu (Core Facilities for Protein Sciences, Chinese Academy of Sciences) for their group's help in maintaining the instruments of our lab.

This work was supported by grants from the National Basic Research Program (973 Program) (Nos. 2011CB910301, 2011CB910900, and 2006CB806506) and the National Natural Science Foundation of China (Grant No. 31021062).

\section{ABBREVIATIONS}

A. suum, Ascaris suum; AA5, atpenin A5; ADI, acceptable daily intake; CBX, carboxin; CHT, ceramic hydroxyapatite; CoQ10, coenzyme Q10; CybL, large cytochrome b binding protein; CybS, small cytochrome b binding protein; DCIP, 2,6-dichlorophenolindophenol; Fp, flavoprotein; HQNO, 2-heptyl-4-hydroxyquinoline-Noxide; $I C_{50}$, half maximal inhibitory concentration; Ip, iron-sulfur protein; MRT, morantel citrate; OAA, oxaloacetate; OXL, oxantel pamoate; PCP, pentachlorophenol; QFR, quinol:fumarate oxidoreductase; SQR, succinate:ubiquinone oxidoreductase; TBZ, thiabendazole; TTFA, a-thenoyltrifluoroacetone; UQ2, 2,3-dimethoxy-5methyl-6-geranyl-1,4-benzoquinone

Supplementary material is available in the online version of this article at http://dx.doi.org/10.1007/s13238-011-1079-1 and is accessible for authorized users.

\section{REFERENCES}

Afanas'eva, E.V., and Kostyrko, V.A. (1986). Pentachlorophenol inhibition of succinate oxidation by the respiratory chain in submitochondrial particles from the bovine heart. Biokhimiia 51, 823-829.

Allen, P.M., and Gottlieb, D. (1970). Mechanism of action of the fungicide thiabendazole, 2-(4'-thiazolyl) benzimidazole. Appl Microbiol 20, 919-926.

Ames, E.R., Cheney, J.M., and Rubin, R. (1963). The efficacy of thiabendazole and bephenium hydroxynaphthoate against Ostertagia ostertagi and Cooperia oncophora in experimentally infected calves. Am J Vet Res 24, 295-299.

Amino, H., Osanai, A., Miyadera, H., Shinjyo, N., Tomitsuka, E., Taka, H., Mineki, R., Murayama, K., Takamiya, S., Aoki, T., et al. (2003). Isolation and characterization of the stage-specific cytochrome $b$ small subunit (CybS) of Ascaris suum complex II from the aerobic respiratory chain of larval mitochondria. Mol Biochem Parasitol 128, 175-186.

Amino, H., Wang, H., Hirawake, H., Saruta, F., Mizuchi, D., Mineki, R., Shindo, N., Murayama, K., Takamiya, S., Aoki, T., et al. (2000). Stage-specific isoforms of Ascaris suum complex. II: The fumarate reductase of the parasitic adult and the succinate dehydrogenase of free-living larvae share a common iron-sulfur subunit. Mol Biochem Parasitol 106, 63-76.

Armson, A., Grubb, W.B., and Mendis, A.H. (1993). Strongyloides ratti: fumarate reductase and succinate dehydrogenase activities of infective larvae. Int J Parasitol 23, 809-811.

Armson, A., Grubb, W.B., and Mendis, A.H. (1995). The effect of electron transport (ET) inhibitors and thiabendazole on the fumarate reductase $(\mathrm{FR})$ and succinate dehydrogenase $(\mathrm{SDH})$ of Strongyloides ratti infective (L3) larvae. Int J Parasitol 25, 261263.

Bion, E., Pariente, E.A., and Maitre, F. (1995). Severe cholestasis and sicca syndrome after thiabendazole. J Hepatol 23, 762-763.

Brown, H.D., Matzuk, A.R., Lives, I.R., and Peterson, L.H.H.,S.A., Sarett, L.H., Egerton, J.R., Yakstis, J.J., Campbell, W.C., and Cuckler, A.C. (1961). Antiparasitic drugs, IV.2-(4'-thiazolyl)benzimidazole, a new anthelmintic. J Am Chem Soc 83, 1764-1765.

Cecchini, G., Maklashina, E., Yankovskaya, V., Iverson, T.M., and Iwata, S. (2003). Variation in proton donor/acceptor pathways in succinate:quinone oxidoreductases. FEBS Lett 545, 31-38.

Cuckler, A.C. (1961). Thiabendazole, a new broad spectrum anthelmintic. J Parasitol 47, 36-37.

DeLano, W.L. (2002). The PyMOL Molecular Graphics System on World Wide Web http://www.pymol.org.

Emsley, P., and Cowtan, K. (2004). Coot: model-building tools for molecular graphics. Acta Crystallogr D Biol Crystallogr 60, 2126-2132.

Eswar, N., Webb, B., Marti-Renom, M.A., Madhusudhan, M.S., Eramian, D., Shen, M.Y., Pieper, U., and Sali, A. (2006). Comparative protein structure modeling using Modeller. Curr Protoc Bioinformatics Chapter 5, Unit 56.

Franklin, R.E., and Gosling, R.G. (1953). Evidence for 2-chain helix in crystalline structure of sodium deoxyribonucleate. Nature 172, 156-157.

Golden, T.A., McElveen, F.J., Jupia, J.E., and Lejeune, C. (1974). Letter: Hematuria following thiabendazole. Arch Dermatol 110, 295.

Gouet, P., Courcelle, E., Stuart, D.I., and Métoz, F. (1999). ESPript: analysis of multiple sequence alignments in PostScript. Bioinformatics 15, 305-308.

Hägerhäll, C. (1997). Succinate: quinone oxidoreductases. Variations on a conserved theme. Biochim Biophys Acta 1320, 107-141.

Horsefield, R., Yankovskaya, V., Sexton, G., Whittingham, W., Shiomi, K., Omura, S., Byrne, B., Cecchini, G., and Iwata, S. (2006). Structural and computational analysis of the quinonebinding site of complex II (succinate-ubiquinone oxidoreductase): a mechanism of electron transfer and proton conduction during ubiquinone reduction. J Biol Chem 281, 7309-7316.

Huang, L.S., Sun, G., Cobessi, D., Wang, A.C., Shen, J.T., Tung, E.Y., Anderson, V.E., and Berry, E.A. (2006). 3-nitropropionic acid is a suicide inhibitor of mitochondrial respiration that, upon oxidation by complex II, forms a covalent adduct with a catalytic base arginine in the active site of the enzyme. J Biol Chem 281, 5965-5972.

Huo, X., Su, D., Wang, A., Zhai, Y., Xu, J., Li, X., Bartlam, M., Sun, F., and Rao, Z. (2007). Preliminary molecular characterization and crystallization of mitochondrial respiratory complex II from porcine heart. FEBS J 274, 1524-1529.

Igual-Adell, R., Oltra-Alcaraz, C., Soler-Company, E., Sánchez- 
Sánchez, P., Matogo-Oyana, J., and Rodríguez-Calabuig, D. (2004). Efficacy and safety of ivermectin and thiabendazole in the treatment of strongyloidiasis. Expert Opin Pharmacother 5, 2615-2619.

Iverson, T.M., Luna-Chavez, C., Cecchini, G., and Rees, D.C. (1999). Structure of the Escherichia coli fumarate reductase respiratory complex. Science, 284, 1961-1966.

Iverson, T.M., Luna-Chavez, C., Croal, L.R., Cecchini, G., and Rees, D.C. (2002). Crystallographic studies of the Escherichia coli quinolfumarate reductase with inhibitors bound to the quinol-binding site. J Biol Chem 277, 16124-16130.

Iwata, F., Shinjyo, N., Amino, H., Sakamoto, K., Islam, M.K., Tsuji, N., and Kita, K. (2008). Change of subunit composition of mitochondrial complex II (succinate-ubiquinone reductase/quinol-fumarate reductase) in Ascaris suum during the migration in the experimental host. Parasitol Int 57, 54-61.

John, C., and Denis, J. (1981). Succinate dehydrogenase and fumarate reductase activity in Aspoculuris Tetraptera and Ascaris Suum and the effect of the anthelmintics cambendazole, thiabendazole, and levamisole. Int J Parasitol 11, 79-84.

Kita, K., Hirawake, H., Miyadera, H., Amino, H., and Takeo, S. (2002). Role of complex II in anaerobic respiration of the parasite mitochondria from Ascaris suum and Plasmodium falciparum. Biochim Biophys Acta 1553, 123-139.

Kita, K., Vibat, C.R., Meinhardt, S., Guest, J.R., and Gennis, R.B. (1989). One-step purification from Escherichia coli of complex II (succinate: ubiquinone oxidoreductase) associated with succinatereducible cytochrome b556. J Biol Chem 264, 2672-2677.

Kuramochi, T., Hirawake, H., Kojima, S., Takamiya, S., Furushima, R., Aoki, T., Komuniecki, R., and Kita, K. (1994). Sequence comparison between the flavoprotein subunit of the fumarate reductase (complex II) of the anaerobic parasitic nematode, Ascaris suum and the succinate dehydrogenase of the aerobic, free-living nematode, Caenorhabditis elegans. Mol Biochem Parasitol 68, 177-187.

Lancaster, C.R., Kröger, A., Auer, M., and Michel, H. (1999). Structure of fumarate reductase from Wolinella succinogenes at 2.2 A resolution. Nature 402, 377-385.

Laskowski, R.A., MacArthur, M.W., Moss, D.S., and Thornton, J.M. (1993). PROCHECK: a program to check the stereochemical quality of protein structures. J Appl Cryst 26, 283-291.

Maklashina, E., and Cecchini, G. (1999). Comparison of catalytic activity and inhibitors of quinone reactions of succinate dehydrogenase (Succinate-ubiquinone oxidoreductase) and fumarate reductase (Menaquinol-fumarate oxidoreductase) from Escherichia coli. Arch Biochem Biophys 369, 223-232.

Maklashina, E., and Cecchini, G. (2010). The quinone-binding and catalytic site of complex II. Biochim Biophys Acta 1797, 1877-1882.

Maklashina, E., Hellwig, P., Rothery, R.A., Kotlyar, V., Sher, Y., Weiner, J.H., and Cecchini, G. (2006). Differences in protonation of ubiquinone and menaquinone in fumarate reductase from Escherichia coli. J Biol Chem 281, 26655-26664.

Manivel, J.C., Bloomer, J.R., and Snover, D.C. (1987). Progressive bile duct injury after thiabendazole administration. Gastroenterology 93, 245-249.

Matsson, M., and Hederstedt, L. (2001). The carboxin-binding site on Paracoccus denitrificans succinate:quinone reductase identified by mutations. J Bioenerg Biomembr 33, 99-105.

McCoy, A.J., Grosse-Kunstleve, R.W., Storoni, L.C., and Read, R.J. (2005). Likelihood-enhanced fast translation functions. Acta Crystallogr D Biol Crystallogr 61, 458-464.

Mileni, M., MacMillan, F., Tziatzios, C., Zwicker, K., Haas, A.H., Mäntele, W., Simon, J., and Lancaster, C.R. (2006). Heterologous production in Wolinella succinogenes and characterization of the quinol:fumarate reductase enzymes from Helicobacter pylori and Campylobacter jejuni. Biochem J 395, 191-201.

Miyadera, H., Shiomi, K., Ui, H., Yamaguchi, Y., Masuma, R., Tomoda, H., Miyoshi, H., Osanai, A., Kita, K., and Omura, S. (2003). Atpenins, potent and specific inhibitors of mitochondrial complex II (succinate-ubiquinone oxidoreductase). Proc Natl Acad Sci U S A 100, 473-477.

Morris, G.M., Huey, R., Lindstrom, W., Sanner, M.F., Belew, R.K., Goodsell, D.S., and Olson, A.J. (2009). AutoDock4 and AutoDockTools4: Automated docking with selective receptor flexibility. J Comput Chem 30, 2785-2791.

Murshudov, G.N., Vagin, A.A., and Dodson, E.J. (1997). Refinement of macromolecular structures by the maximum-likelihood method. Acta Crystallogr D Biol Crystallogr 53, 240-255.

Ogata, A., Ando, H., Kubo, Y., and Hiraga, K. (1984). Teratogenicity of thiabendazole in ICR mice. Food Chem Toxicol 22, 509-520.

Otwinowski, Z., and Minor, W. (1997). Processing of X-ray diffraction data collected in the oscillation mode. Methods Enzymol 276, 307-326.

Pawlowski, Z.S., and Skrzypinska, K. (1972). Urine precipitates during thiabendazole treatment. Trans R Soc Trop Med Hyg 66, 512.

Portugal, R., Schaffel, R., Almeida, L., Spector, N., and Nucci, M. (2002). Thiabendazole for the prophylaxis of strongyloidiasis in immunosuppressed patients with hematological diseases: a randomized double-blind placebo-controlled study. Haematologica 87, 663-664.

Ramsay, R.R., Ackrell, B.A., Coles, C.J., Singer, T.P., White, G.A., and Thorn, G.D. (1981). Reaction site of carboxanilides and of thenoyltrifluoroacetone in complex II. Proc Natl Acad Sci U S A 78, 825-828.

Rex, D., Lumeng, L., Eble, J., and Rex, L. (1983). Intrahepatic cholestasis and sicca complex after thiabendazole. Report of a case and review of the literature. Gastroenterology 85, 718-721.

Robinson, H.J., Phares, H.F., and Graessle, O.E. (1978). The toxicological and antifungal properties of thiabendazole. Ecotoxicol Environ Saf 1, 471-476.

Robinson, H.J., Stoerk, H.C., and Graessle, O.E. (1965). Studies on the toxicologic and pharmacologic properties of thiabendazole. Toxicol Appl Pharmacol 7, 53-63.

Roy, M.A., Nugent, F.W., and Aretz, H.T. (1989). Micronodular cirrhosis after thiabendazole. Dig Dis Sci 34, 938-941.

Ruprecht, J., Yankovskaya, V., Maklashina, E., Iwata, S., and Cecchini, G. (2009). Structure of Escherichia coli succinate: quinone oxidoreductase with an occupied and empty quinonebinding site. J Biol Chem 284, 29836-29846.

Saraste, M. (1999). Oxidative phosphorylation at the fin de siecle. Science 283, 1488-1493.

Stoscheck, C.M. (1990). Quantitation of protein. Methods Enzymol 182, 50-68.

Strong, M., Sawaya, M.R., Wang, S., Phillips, M., Cascio, D., and 
Eisenberg, D. (2006). Toward the structural genomics of complexes: crystal structure of a PE/PPE protein complex from Mycobacterium tuberculosis. Proc Natl Acad Sci U S A 103, 8060-8065.

Sun, F., Huo, X., Zhai, Y., Wang, A., Xu, J., Su, D., Bartlam, M., and Rao, Z. (2005). Crystal structure of mitochondrial respiratory membrane protein complex II. Cell 121, 1043-1057.

Tada, Y., Fujitani, T., Yano, N., Yuzawa, K., Nagasawa, A., Aoki, N., Ogata, A., and Yoneyama, M. (2001). Chronic toxicity of thiabendazole (TBZ) in CD-1 mice. Toxicology 169, 163-176.

Thompson, J.D., Higgins, D.G., and Gibson, T.J. (1994). CLUSTAL W: improving the sensitivity of progressive multiple sequence alignment through sequence weighting, position-specific gap penalties and weight matrix choice. Nucleic Acids Res 22, 4673-4680.

Yankovskaya, V., Horsefield, R., Tornroth, S., Luna-Chavez, C., Miyoshi, H., Leger, C., Byrne, B., Cecchini, G., and Iwata, S. (2003). Architecture of succinate dehydrogenase and reactive oxygen species generation. Science 299, 700-704.

Yankovskaya, V., Sablin, S.O., Ramsay, R.R., Singer, T.P., Ackrell, B. A., Cecchini, G., and Miyoshi, H. (1996). Inhibitor probes of the quinone binding sites of mammalian complex II and Escherichia coli fumarate reductase. J Biol Chem 271, 21020-21024. 